\title{
Notfall Psyche - Diagnostik und Krisenintervention in der Präklinik
}

Wolfgang Jordan

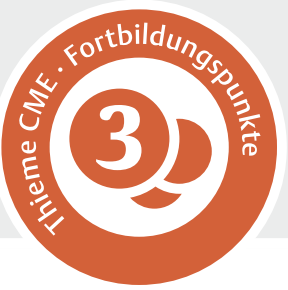

Der psychiatrische Notfall gehört zu den häufigsten Einsatzursachen für Notärzte. Der Beitrag bietet einen Leitfaden zum Umgang mit Menschen in seelischer Not: von der Orientierung im Notfallgeschehen über eine Zusammenstellung der häufigsten Krankheitsbilder, die Vorstellung spezieller Techniken zur Krisenintervention bis hin zu pharmakologischen Aspekten.

\begin{tabular}{|c|c|}
\hline \multicolumn{2}{|c|}{ ABKÜRZUNGEN } \\
\hline COPD & chronisch obstruktive Lungenerkrankung \\
\hline EPMS & extrapyramidalmotorische Störung \\
\hline LSD & Lysergsäurediethylamid \\
\hline $\mathrm{MAOH}$ & Monoaminoxidasehemmer \\
\hline MDA & 3,4-Methylendioxyamphetamin (Eve) \\
\hline MDMA & $\begin{array}{l}\text { 3,4-Methylendioxymetamphetamin } \\
\text { (Ecstasy) }\end{array}$ \\
\hline NaSSA & $\begin{array}{l}\text { noradrenerg/spezifisch serotonerges } \\
\text { Antidepressivum mit } \alpha_{2} \text {-Adrenozeptor- } \\
\text { antagonistischer Wirkung }\end{array}$ \\
\hline PsychKG & Psychisch-Kranken-Gesetz \\
\hline SSNRI & $\begin{array}{l}\text { selektiver Serotonin- und Noradrenalin- } \\
\text { wiederaufnahmehemmer }\end{array}$ \\
\hline SSRI & $\begin{array}{l}\text { selektiver Serotoninwiederaufnahme- } \\
\text { hemmer }\end{array}$ \\
\hline TZA & trizyklisches Antidepressivum \\
\hline TZ-NL & trizyklisches Neuroleptikum \\
\hline ZNS & zentrales Nervensystem \\
\hline
\end{tabular}

\section{Einleitung}

Psychiatrische Notfälle sind in der präklinischen Notfallmedizin so häufig wie traumatologische und neurologische Notfälle. Sie gehören mit 10-15\% zu den häufigsten Einsatzursachen für den Notarzt [1,2].

Der Notfall „Psyche“ ist allgegenwärtig. Er findet sich bei

- psychischen oder psychiatrischen Störungen,

- psychischen Ausnahmereaktionen auf belastend empfundene Lebensereignisse, u. a. Schicksalsschläge,

- oder als extreme psychische Begleitreaktion bei akut einsetzenden körperlichen Beeinträchtigungen im eigenen oder im Erleben emotional nahestehender Dritter wie z. B.

- Apoplex,

- Angina pectoris,
- Dyspnoe,

- Asthmaanfall,

- Koliken,

- Harnverhalt,

- Glaukomanfall,

- Verbrennungen,

- Frakturen.

\section{Merke}

Der Ersthelfer ist in jeder Notfallsituation gefordert, somatische und psychische Belange gleichzeitig zu erfassen, zu bewerten und sein weiteres Vorgehen darauf abzustimmen. Ansonsten besteht die Gefahr einer wechselseitigen Verstärkung, einer Kollusion, die jede Krise zur Eskalation bringen kann.

Psychiatrische Notfälle stellen für die im Rettungsdienst Tätigen selbst eine schwere psychische Belastung dar [3, 4]. Das eigene Risiko für eine posttraumatische Belastungsstörung oder eine Depression beträgt rund 10\%, wobei eine vorbestehende psychiatrische Störung das Risiko um das 5- bis 6-Fache erhöht [5]. Die Beachtung und Anwendung spezifischer Techniken trägt zum Selbstschutz bei [6].

\section{LERNZIELE}

Nach dem Lesen des Beitrags haben Sie Kenntnisse

- zur Methodik der psychiatrischen Notfall- und Krisenintervention,

- zur Diagnosestellung psychiatrischer Notfälle,

- zur Notfallpsychopharmakologie,

- zu den wichtigsten Syndromen psychiatrischer Notfälle,

- einschließlich der zugehörigen Krisenintervention. 


\section{Allgemeine Notfallpsychiatrie}

\section{Methodik der psychiatrischen Notfall- und Krisenintervention}

Psychiatrisch-psychotherapeutische Notfälle können mit Selbst- und/oder Fremdgefährdung einhergehen, was bei einem professionellen Vorgehen unbedingt zu berücksichtigen ist. Vor dem ersten Kontakt sollte eine Risikoabschätzung vorgenommen werden:

\section{Wie wurde das Krisengeschehen über die Rettungs-} kette an die Rettungskräfte herangetragen?

Aus der Beantwortung der Frage lassen sich mögliche Rückschlüsse auf die mit dem Störungsbild verbundene Psychodynamik und Beziehungsgestaltung zum sozialen Umfeld ziehen. Je akuter eine krisenhafte Entwicklung stattgefunden hat, umso höher sollte das Fremdgefährdungspotenzial eingeschätzt werden. Das Hinzuziehen Professioneller ist als Zeichen einer Eskalation zu bewerten, nachdem die stützenden Maßnahmen im sozialen Umfeld nicht mehr gegriffen haben [6]. Gibt es ein Netzwerk zuverlässiger, erreichbarer, wertschätzender und hilfsbereiter Personen, auf die als stabilisierende Ressource zurückgegriffen werden kann? Oder ist eher mit einer Verschlimmerung der Situation zu rechnen, weil ein möglicher Gesprächspartner direkt in der auslösenden Situation involviert war oder ein Eigeninteresse an einer krisenhaften Zuspitzung hat? Bei den vorgesehenen Gesprächspartnern ist eine ausreichende Motivation, Belastbarkeit und Kommunikationsfähigkeit zu prüfen [6].

\section{Fremdinformationen eruierbar?}

Gibt es Fremdinformationen zu dem Betroffenen, zu beteiligten Personen, zu dem Ablauf des Geschehens, zu der aufzusuchenden Örtlichkeit?

Gegebenenfalls lassen sich zufällig anwesende Dritte schnell befragen. Mit dem Betreten der Örtlichkeit der Krisenintervention, z.B. der Wohnung des Betroffenen, sollten Umfeldinformationen aus der Sichtung der Zimmer, insbesondere eigener Platz, Bett, Nachttisch, persönliche Bilder, Bücher, Fotos, Medikamente, Drogenutensilien usw., Berücksichtigung finden [6]. Gibt es Hinweise auf abgelaufene Gewalt, z.B. verängstigte Personen oder eine beschädigte Einrichtung?

\section{Merke \\ Die Rahmenbedingungen bestimmen das Vorgehen im Notfall.}

Seelische Notzustände können viele Ursachen haben. Trotzdem lassen sich häufig einheitliche Reaktionsformen oder Muster erkennen [6] (s. Infobox).

\section{ÜBERSICHT}

\section{Reaktionsformen/-muster im Verhalten bei} psychiatrischen Notfällen

- depressive oder missmutig-aggressive Stimmung, Affektlabilität mit anlassbedingtem schnellem Stimmungswechsel, psychomotorisches und vegetatives Hyperarousal

- Einengung der Wahrnehmung auf die Themen Kränkung und Verlust, Schwarz-Weiß-Denken, interne Kommunikationsstörung mit nachfolgender Missdeutung der beteiligten Kommunikationspartner

- starres Rollenverhalten, Einnahme einer Patientenrolle, unflexibles Problemlöseverhalten

- sozialer Rückzug und/oder Rückzug des sozialen Umfeldes

\section{Rahmenbedingungen}

Die Beziehungsgestaltung und das Arrangement des Settings hängen von den krisen- und krankheitsbedingten Erfordernissen ab. Dabei ist zu berücksichtigen, dass im Rahmen der psychischen Störung Veränderungen eingetreten sein können betreffend

- die Bewusstseinslage (quantitativ und qualitativ),

- das Orientierungsvermögen (zeitlich, örtlich, situativ, zur Person),

- die Aufmerksamkeit,

- die Konzentration, Ablenkbarkeit, Merkfähigkeit,

- das formale Denken (Störungen des Gedankenablaufs, z.B. Verlangsamung, Hemmung, Einengung, Umständlichkeit, Weitschweifigkeit, Ideenflucht, Zerfahrenheit),

- das inhaltliche Denken (z. B. Wahn),

- Affekte,

- Psychomotorik;

- oder die Betroffenen können im paranoid-halluzinatorischen Erleben befangen sein.

Die Örtlichkeit der Krisenintervention, z. B. ein bestimmtes Zimmer in einer Wohnung, ist entsprechend der vorbekannten, ersichtlichen oder zu erwartenden Beeinträchtigung auszuwählen und ggf. an die Erfordernisse anzupassen.

\section{PRAKTISCHER HINWEIS}

Dabei hat die Berücksichtigung der persönlichen Sicherheit die höchste Priorität. Gibt es eine Fluchtmöglichkeit, kann schnell Hilfe gerufen werden, befinden sich gefährliche Gegenstände im Raum oder in unmittelbarer Nähe des Betroffenen (z. B. Messer, Brieföffner, Schere, Glasflasche, Stuhl usw.), die als Waffe verwendet werden können? 
Merke

Immer auf die persönliche Sicherheit achten!

Durch die Anordnung der Sitzmöglichkeiten und Ausrichtung der Blickrichtung kann eine Privatsphäre geschaffen, eine Reizabschirmung vorgenommen und eine Fokussierung auf den Behandler vereinfacht werden. Die genannten Maßnahmen mögen seltsam erscheinen, tragen aber zu einer Verbesserung des Beziehungsaufbaus und der -gestaltung bei.

Ein ausreichendes Zeitmanagement sichert dem Behandler die innere Gelassenheit und führt zur Beruhigung der Krisensituation. Seine primäre Aufgabe im Erstkontakt besteht darin, die Situation zu klären und - falls erforderlich - den Hilfesuchenden sicher zur weiterführenden Therapie zu bringen. Es ist nicht Aufgabe im Erstkontakt, die Probleme eines ganzen fehlgelaufenen Lebens zu lösen. Das Vorgehen sollte somit lösungsorientiert auf den primären Ansatz hin sein.

Ablauf

Mit Sprache, Mimik und Gestik ist ein Setting zu schaffen, in dem sich der Betroffene mit seinen Sorgen angenommen, vorurteils- und angstfrei äußern kann. Die Grundhaltung des Behandlers ist von Empathie (einfühlendem Verständnis), positiver Wertschätzung (Akzeptanz, emotionaler Wärme) und Authentizität (Echtheit) geprägt, im weiteren Vorgehen aber deutlich direktiver. Der Gesprächsverlauf wird zu Beginn von dem Betroffenen bestimmt, um einen unverfälschten diagnostischen Eindruck zu bekommen und eine erste Entlastung zu erreichen. In der Folge wird der Behandler aktiv intervenieren und den weiteren Ablauf steuern.

Das Gespräch verfolgt einen diagnostischen, situationsklärenden und einen therapeutischen, problemlösenden Zweck. Je nach psychischem Befund kann im diagnostischen Ansatz über eine kurzzeitige, wenn möglich körpersprachlich unterlegte Intervention des Behandlers das Erregungsund Aggressionspotenzial des Betroffenen ausgetestet werden. Diese sollte aber unmittelbar danach zurückgenommen werden, um den weiteren Beziehungsaufbau nicht zu gefährden und zur Eskalation der Situation beizutragen.

\footnotetext{
Merke

Die eigene verbale und nonverbale Kommunikationsfähigkeit (Körpersprache) stellen das wichtigste Interventionsinstrument dar. Die gewählte Sprache sollte klar, einfach und verständlich sein sowie ausreichend Wiederholungen und Zusammenfassungen beinhalten. Die über die Körpersprache vermittelten Signale sollten mit der geäußerten Botschaft übereinstimmen und sie ggf. noch bekräftigen [6].
}

Der Ablauf einer Notfall- und Krisenintervention wird stark von den aktuellen Umständen und den Gegeben- heiten vor Ort geprägt. Trotzdem gibt es ein gemeinsames Vorgehen und einheitliche Regeln, deren Beachtung für die Lösung der Situation förderlich ist [6]:

- Sich selbst vorstellen!

- Betroffene mit berichtetem, ersichtlichem, getestetem (s.o.) oder spürbarem Aggressionspotenzial nie allein sprechen oder untersuchen!

- Setting herstellen und gestalten (s.o.).

- Zuhören und offene W-Fragen ohne vorgegebene Antworten zu Beginn, im Verlauf direktiveres Vorgehen mit Übernahme der Gesprächsführung.

- Ansprechen von Gefühlen und Ambivalenz, ggf. auch Ansprechen eigener Gefühle.

\section{FALLBEISPIEL}

\section{Ambivalenz ansprechen und lösen}

„Herr Mustermann, ich sehe an Ihrer Körperhaltung, wie Sie sich sorgen und nicht in das Krankenhaus wollen. Das geht jetzt leider nicht anders. Ich nehme Sie mit. Kommen Sie bitte.“

\section{FALLBEISPIEL}

\section{Ansprechen eigener Gefühle}

„Herr Mustermann, Sie machen mir Angst! Wie können Sie sich das erklären?"

- Genaues Klären der Situation und wirkliches Verstehen! Der Behandler gibt sich nicht mit vermeintlich einfachen oder vordergründigen Erklärungen zufrieden, sondern versucht, durch gezieltes Nachfragen die äußeren und inneren Zusammenhänge wirklich zu verstehen. Oft ist es wegweisend, die Bedeutungszusammenhänge aus Betroffenensicht zu erfragen:

\section{FALLBEISPIEL}

Bedeutungszusammenhänge aus Betroffenensicht erfragen

„Herr Mustermann, was denken Sie, ist die Ursache Ihrer Probleme?“

Oder alternativ, wenn der Betroffene aus der eigenen Perspektive nicht antworten möchte oder kann: „Herr Mustermann, was sagt Ihre Frau, Ihr ... zu dem Vorfall?“

Zur weiteren Klärung kann eine Plausibilitätskontrolle erfolgen, ob der Behandler oder eine ihm bekannte Person in einer vergleichbaren Situation ebenso reagiert hätte. In diesem Zusammenhang sollten auch die Motivation und Veränderungsbereitschaft des Betroffenen geklärt werden. 
- Behandlungsauftrag klären - aus Sicht des Betroffenen, der Angehörigen, des Behandlers? - und gemeinsam formulieren. Die Notfallaufgabe ist dabei auf Machbarkeit zu beschränken. Es sollten klare, lösungsorientierte Prioritäten gesetzt und eine realistische Planung vorgenommen werden.

- Einsatz der eigenen Kommunikationskompetenz, eine Beziehung aufzubauen, zu gestalten und den Hilfesuchenden und seine Angehörigen im Gespräch zu führen unter Berücksichtigung unterschiedlicher therapeutischer Rollen, z. B.

- empathisch,

- entlastend,

- schützend,

- Ich/Selbstwert stärkend,

- Grenzen setzend,

- konfrontativ,

- einengend.

- In Abhängigkeit von der Situation muss der Behandler unterschiedliche Rollen einnehmen können. Diese können während eines Gesprächs durchaus mehrfach wechseln. Bei der Wahl seines Vorgehens sollte er die Kommunikations- und Problemlösekompetenz bei dem Betroffenen und seinem Umfeld beachten.

- Lösungsorientierte, selbstwertstärkende Interventionen! Die Interventionen sollten zu einer Stärkung des positiven Selbstbildes beitragen, Regressionen vermeiden und dem Hilfesuchenden Verantwortung im Rahmen seiner Möglichkeiten übertragen. Bei unzureichender Belastbarkeit und fehlendem innerem Zusammenhalt sollten keine Bewertungen, Interpretationen oder Deutungen vorgenommen werden. Allenfalls positive Muster können vorsichtig zurückgespiegelt werden. Gewünschte Verhaltensweisen sind positiv zu verstärken.

\section{FALLBEISPIEL}

\section{Positive Verstärkung}

„Herr Mustermann, vielen Dank, dass Sie den Vorgang so detailliert geschildert haben. Das war jetzt sehr hilfreich für mich, um die Situation richtig zu verstehen.“

\section{Merke}

Bei jeder Intervention wird der konstruktive Anteil der Persönlichkeit gefördert, wobei das zerstörerische Potenzial unbedingt zu berücksichtigen ist.

- Planung realistischer Veränderungen, eindeutig, zeitlich begrenzt, unter Einbindung des Hilfesuchenden, sofern möglich mit Beteiligung der Angehörigen, möglichst verbindlich.

- Maßnahmen bei Überforderung: Die Aktivierung von Betroffenen und Angehörigen, die Verschiebung des Fokus, z. B. durch Fragen zur Person, anerkennende
Äußerungen zu Kleidung, Frisur oder Schmuck, Fragen nach Haustieren bei Alleinstehenden oder Hobbys, wenn sie aus dem Kontext erschließbar sind, und die Umlenkung des Affektes, etwa durch Humor, sind hilfreiche Maßnahmen bei Überforderung. Sie führen zu einem Wechsel der Perspektive und erhöhen die innere Distanz zum Geschehen.

- Störungen durch Dritte: „Engagierte“ Angehörige oder störende Dritte, die mit ihrem hektischen Verhalten eher zur Beunruhigung aller beitragen, sollten mit einer „wichtigen“ Aufgabe betraut werden, z. B. Tee machen, sich um das Haustier kümmern, dem Rettungsdienst den Weg weisen oder Ähnliches.

- Der Verabschiedung des Hilfesuchenden und seiner Angehörigen ist ausreichend Zeit einzuräumen, sodass die im Gespräch aufgebaute Entspannung mitgenommen werden kann. Da Konzentration und Aufmerksamkeit aller an einer Notfallsituation Beteiligten beeinträchtigt sein können, empfiehlt es sich, zum Abschluss jedes Gesprächs Rückfragen zur „Ergebnissicherung" vorzunehmen und ggf. die wichtigsten Festlegungen nochmals zu wiederholen. Dabei sollte der Patient zu einer weiterführenden Bearbeitung seines Problems - am besten unter Benennung von Anlaufstellen - ermuntert werden. Gegebenenfalls ist die Verabschiedung auch zur Betonung bzw. zum Aufbau von Zukunftsbezügen zu nutzen [6].

\section{Diagnosestellung}

Die Umstände und das Störungsbild in der psychiatrischen Notfallsituation werden oft nur eine eingeschränkte Untersuchung des Betreffenden zulassen. Die Anamneseerhebung wird dann auf die aktuellen Hauptbeschwerden und die Fremdanamnese begrenzt sein, und auch eine klinisch-neurologische Untersuchung wird sich gerade bei Patienten mit psychomotorischer Erregung zunächst auf das Augenscheinliche, das Wesentliche beschränken müssen [6].

\section{FALLBEISPIEL}

Neurologische „Untersuchung“ eines psychomotorisch erregten Patienten im häuslichen Umfeld.

Der Notarzt wird zu einem psychomotorisch erregten Patienten gerufen. Der Patient begrüßt den Notarzt und Rettungssanitäter mit einem rechten Schwinger, den der Arzt kommentiert: „Schau, den rechten Arm kann er schon bewegen. “ Der Arzt muss einem Schwinger links ausweichen und führt fort: „Beide obere Extremitäten frei beweglich, Koordination leicht eingeschränkt. Jetzt muss ich mich noch beidseits treten lassen, dann ist die Prüfung der Motorik abgeschlossen.“ 
- Tab. 1 Pathognomische Psychopathologie.

\begin{tabular}{|c|c|}
\hline Symptom & Syndrom, Diagnose \\
\hline \multicolumn{2}{|l|}{ formale Denkstörungen } \\
\hline $\begin{array}{l}\text { Ideenflucht (viele Themen, aber Assoziationen erkennbar und } \\
\text { Gedankengang nachvollziehbar) }\end{array}$ & Manie \\
\hline $\begin{array}{l}\text { Zerfahrenheit, Inkohärenz (Assoziationen oft nicht mehr erkennbar, } \\
\text { Gedankengang kaum oder nicht mehr nachvollziehbar) }\end{array}$ & $\begin{array}{l}\text { Schizophrenie } \\
\text { hirnorganisch }\end{array}$ \\
\hline $\begin{array}{l}\text { Weitschweifigkeit (fehlende Trennung wesentlich - unwesentlich im } \\
\text { Gedankengang) }\end{array}$ & $\begin{array}{l}\text { hirnorganisch } \\
\text { Persönlichkeitsstörung }\end{array}$ \\
\hline \multicolumn{2}{|l|}{ inhaltliche Denkstörungen (Wahn) } \\
\hline Verarmungs-, Versündigungs-, Schuldwahn & schwere Depression \\
\hline Größenwahn & $\begin{array}{l}\text { Manie } \\
\text { Schizophrenie }\end{array}$ \\
\hline Beziehungs-, Beeinträchtigungs-, Verfolgungswahn & Schizophrenie \\
\hline Dermatozoenwahn & hirnorganisch \\
\hline Eifersuchtswahn & chronische Alkoholabhängigkeit \\
\hline \multicolumn{2}{|l|}{ Affektstörungen } \\
\hline Parathymie (Diskrepanz geäußerter Inhalt und Affekt) & Schizophrenie \\
\hline $\begin{array}{l}\text { Affektlabilität (schneller Stimmungswechsel durch äußeren Anstoß } \\
\text { oder innerer Beschäftigung mit emotionalem Thema) }\end{array}$ & $\begin{array}{l}\text { unspezifisch } \\
\text { psycholabile Menschen } \\
\text { bipolare Störungen } \\
\text { Depression } \\
\text { Demenz }\end{array}$ \\
\hline $\begin{array}{l}\text { Affektinkontinenz (überschießender nicht beherrschbarer Affekt, z. B. } \\
\text { unvermitteltes Weinen ohne klaren thematischen Bezug) }\end{array}$ & hirnorganisch, Demenz \\
\hline \multicolumn{2}{|l|}{ Wahrnehmungsstörungen } \\
\hline akustische Halluzinationen & $\begin{array}{l}\text { unspezifisch, z. B. paranoid-halluzinatorische Psychose, } \\
\text { Alkoholhalluzinose, Schizophrenie }\end{array}$ \\
\hline optische Halluzinationen & organisch, auch Delir, medikamentös \\
\hline gustatorische Halluzinationen & Epilepsie \\
\hline \multicolumn{2}{|l|}{ Ichstörungen } \\
\hline Depersonalisation, Derealisation & $\begin{array}{l}\text { unspezifisch, z. B. auch im normalen Erleben oder bei Persön- } \\
\text { lichkeitsstörungen, posttraumatischen Belastungsstörungen }\end{array}$ \\
\hline Gedankeneingebung, -entzug, -ausbreitung, Willensbeeinflussung & Schizophrenie \\
\hline
\end{tabular}

Trotz der eingeschränkten Untersuchungsbedingungen lassen sich aus einer sorgsamen Inspektion und Beobachtung des Patienten zahlreiche diagnostisch wichtige Rückschlüsse ziehen, beispielsweise

- äußeres Erscheinungsbild:

- Art der Kleidung,

- Körperschmuck,

- Hinweis auf Gewalttätigkeiten,

- Verletzungen,

- Einstichstellen,

- vegetative Elementarfunktionen:

- Atmung, Puls

- Hautfarbe und -turgor,

- Pupillenweite,
- Hyperhidrosis,

- Tremor.

Für die Diagnosestellung sind sämtlich beobachtbare Auffälligkeiten im Verhalten und Erleben des Betreffenden gleichwertig dem gesprochenen Wort. Das eigene Erleben, die Reaktion des Behandlers auf den Patienten, ist als ergänzendes diagnostisches Instrument bewusst zu nutzen (sogenannte Übertragung und Gegenübertragung).

Die Kenntnis der Psychopathologie, d. h. die korrekte Erfassung und Benennung psychischer Auffälligkeiten, hilft bei der Abgrenzung und Zuordnung einzelner Störungsbilder ( $\triangleright$ Tab. 1). 
- Tab. 2 Auswahlkriterien bei der psychopharmakologischen Notfallmedikation.

\begin{tabular}{|c|c|}
\hline $\begin{array}{l}\text { Aspekt der } \\
\text { Medikation }\end{array}$ & $\begin{array}{l}\text { Augenmerk bzw. } \\
\text { Auswahlkriterium }\end{array}$ \\
\hline $\begin{array}{l}\text { Notarztwagen, soma- } \\
\text { tische Station? }\end{array}$ & Verfügbarkeit \\
\hline Multimorbidität? & $\begin{array}{l}\text { Interaktionsprofil mit geringen } \\
\text { Wechselwirkungen }\end{array}$ \\
\hline Suizidalität? & hohe therapeutische Breite \\
\hline Pharmakokinetik & $\begin{array}{l}\text { schneller Wirkeintritt } \\
\text { gute Steuerbarkeit }\end{array}$ \\
\hline Nebenwirkungsprofil & $\begin{array}{l}\text { gering } \\
\text { keine besondere Überwachung }\end{array}$ \\
\hline Dosierung & $\begin{array}{l}\text { möglichst einfach, dabei } \\
\text { Therapiedauer beachten! }\end{array}$ \\
\hline
\end{tabular}

Das Ergebnis der Eingangsdiagnostik bestimmt die weitere Beziehungsgestaltung und Krisenintervention einschließlich der Anwendung spezifischer Techniken und damit den guten Ausgang.

Merke

Der gute Ausgang einer Krisenintervention hängt maßgeblich vom diagnostischen Blick des Behandlers ab.

\section{Notfallpsychopharmakotherapie}

Die Auswahl eines geeigneten Medikaments zur Notfallbehandlung hängt von den äußeren Umständen, der aktuellen klinischen Symptomatik sowie möglichen Begleiterkrankungen und -medikation ab ( Tab. 2).

\section{Empfohlene Substanzen}

\section{Aktivierende Antidepressiva}

Falls ein aktivierendes Antidepressivum eingesetzt werden soll, empfiehlt sich Escitalopram (Cipralex ${ }^{\circledR}$ ). Es ist der selektivste Serotoninwiederaufnahmehemmer (SSRI) mit breiter Indikation, guter Verträglichkeit und geringem Interaktionsrisiko.

Wird ein schlafanstoßendes, -unterhaltendes, sedierendes Antidepressivum benötigt, fällt die Wahl auf Mirtazapin (Remergil ${ }^{\circledR}$ ), ein noradrenerg spezifisch serotonerges Antidepressivum mit $\alpha_{2}$-Adrenozeptor-antagonistischer Wirkung (NaSSA). Beide empfohlenen Antidepressiva haben möglicherweise eine etwas bessere Wirkung und einen geringfügig schnelleren Wirkeintritt als andere Antidepressiva. Mirtazapin hat ebenfalls ein geringes Interaktionspotenzial [6].

\section{Klassische Antipsychotika}

Aus der Gruppe der klassischen Antipsychotika werden als hochpotente Substanz Haloperidol $\left(\mathrm{Haldo}^{\circledR}\right)$ und als niederpotente Melperon (Eunerpan ${ }^{\circledR}$ ) empfohlen, beides sind Substanzen mit einer großen Anwendungserfah- rung. Eine breite Zulassung für unterschiedliche psychomotorische Erregungszustände liegt vor. Beide Antipsychotika sind vom Butyrophenon-Derivattyp und wegen der fehlenden anticholinergen Komponente zur Sedierung bei psychomotorischen Erregungszuständen besser geeignet als trizyklische Antipsychotika.

\section{Atypische Antipsychotika}

Von den atypischen Antipsychotika erscheinen Olanzapin (Zyprexa ${ }^{\circledR}$ ) und Amisulprid (Solian ${ }^{\circledR}$ ) ratsam. Olanzapin ist eine sedierende, Amisulprid eine eher nicht sedierende Substanz, beide mit geringem Risiko für extrapyramidalmotorische Störungen (EPMS). Amisulprid wird ohne hepatische Metabolisierung unverändert renal ausgeschieden, sodass kein pharmakokinetisches Interaktionsrisiko besteht. Sowohl Olanzapin als auch Amisulprid haben keine Zulassung zur Behandlung psychomotorischer Erregungszustände außerhalb der schizophrenen und/oder manischen Störung. Olanzapin ist für die Behandlung der Schizophrenie und Manie, Amisulprid allein für die der Schizophrenie zugelassen. Neben der klassischen Tablettenform ist Olanzapin als Schmelztablette und Amisulprid als Lösung (Solian ${ }^{\circledR}$ ) erhältlich [6].

\section{Anxiolytika, Sedativa}

Als Anxiolytikum oder Sedativum wird Lorazepam (Tavor $^{\circledR}$ ) empfohlen. Es ist ein relativ kurzwirksames Benzodiazepin mit einer Halbwertszeit zwischen 12 und $16 \mathrm{~h}$ ohne aktive Metaboliten. Lorazepam kann per os (p. o.), auch als Schmelztablette (Expidet), oder parenteral langsam intravenös (i. v.) oder intramuskulär (i. m.) appliziert werden. Es verfügt über eine stupor- und mutismuslösende Wirkung.

In seltenen Fällen besteht bei gespannt-aggressiven Patienten die Gefahr eines Raptusdurchbruchs, eines unvermittelten Gewaltausbruchs. Hier erscheint ein stärker sedierendes Benzodiazepin wie Diazepam (s. u.) besser geeignet [6].

\section{Häufig eingesetzte, nicht empfohlene Substanzen}

Levomepromazin $\left(\right.$ Neuroci $\left.{ }^{\circledR}\right)$ ist ein niederpotentes trizyklisches Antipsychotikum, welches gerne zur Behandlung psychomotorischer Erregungszustände eingesetzt wird. Wegen seiner ausgeprägten anticholinergen $\mathrm{Ne}$ benwirkungskomponente (Sinustachykardie, Herzrhythmusstörungen, Gedächtnisstörungen, Verwirrtheit, Delir, Akkommodationsstörungen, Mundtrockenheit, Obstipation, Miktionsstörungen) wird es nicht empfohlen. Bei der Anwendung sollten kardiale und zerebrale Vorschädigungen, Glaukom und Prostatahypertrophie ausgeschlossen sein. Üblicherweise werden bei psychiatrischen Notfällen 25-50 mg i.m. appliziert, bei Bedarf kann alle 30-60 min bis maximal $150 \mathrm{mg} / \mathrm{d}$ nachinjiziert werden. Eine höhere initiale Dosis sollte nicht gegeben werden, weil darunter eine orthostatische Dysregulation mit Hypotonie und Kollaps auftreten kann. Bei i. v. Appli- 
kation kann es zu Thrombophlebitiden kommen, weswegen die Substanz nur verdünnt langsam injiziert werden sollte [6].

Diazepam ist das Benzodiazepin mit dem schnellsten Wirkeintritt, was es eigentlich für den Einsatz in der Notfallbehandlung prädestiniert. Diazepam verfügt aber über ein großes Verteilungsvolumen, sodass bei Einmaldosierung die Wirkung nur kurz andauert. Insbesondere bei Mehrfachgabe unter der Vorstellung, dass die Substanz zu gering dosiert wurde, können wegen des hohen Verteilungsvolumens Rückverteilungsphänomene auftreten. Die Substanz wird zeitverzögert wieder aus der Muskulatur und dem Fettgewebe freigesetzt und führt plötzlich zu einer klinischen Überdosierung. Wegen aktiver Metaboliten, die eine Halbwertszeit bis $200 \mathrm{~h}$ aufweisen, besteht zudem eine Kumulationsgefahr. Die gebräuchliche Dosierung ist 5-10 mg p.o., i.v. oder i.m., die ggf. 1-2 $\times$ nach 30 min wiederholt werden kann, bis maximal $40 \mathrm{mg} / \mathrm{d}$. Höhere Dosen können zu einer Atemdepression führen, weswegen auch die i.v. Gabe nur langsam erfolgen sollte [6].

\section{FAZIT}

Die Sicherheit und Erfahrung des Behandlers im Umgang mit dem Medikament ist entscheidender als die benutzte Substanz.

Primäres Behandlungsziel ist Beruhigung und nicht Sedierung. Der Patient soll zwar schnell stabilisiert werden, für den weiterbehandelnden Psychiater aber noch explorierbar sein!

Die Mischung verschiedener Substanzen nach „eigener Rezeptur“ mit z. T. abenteuerlicher Bezeichnung, z. B. lytischer Trunk, hat in der Notfallpsychopharmakotherapie nichts zu suchen!

\section{Selbsthilfe für das medizinische Personal}

Die Achtsamkeit den eigenen Grenzen gegenüber, die klare Begrenzung einer Aufgabe auf ihre Machbarkeit mit paralleler Kommunikation nach außen und die rechtzeitige Hinzuziehung weiterer professioneller Unterstützung (z. B. Polizei, Sozialpsychiatrischer Dienst, Krisendienst, andere Kollegen, eigene Supervision) helfen, eine Überforderung zu vermeiden. In einer für sich selbst belastend empfundenen Situation können Techniken zur Anwendung kommen, welche die therapeutische Distanz vergrößern [6].

\section{PRAXISTIPPS}

\section{Äußere Distanzierung durch Veränderung der Situation}

Eine Änderung der Situation lässt sich räumlich über einen Wechsel der Sitzposition, des Stuhls mit einer neuen Blickausrichtung oder auch den Wechsel in ein anderes Zimmer erreichen. Situativ kann ein anderes Thema eingeführt werden (z. B. Urlaub, Haustiere), der Behandler kann sich die Küche, das Wohnzimmer, die Bibliothek zeigen oder bei dem Patienten eine Blutentnahme oder Blutdruckmessung durchführen lassen.

\section{Innere Distanzierung durch Perspektivenwechsel und Fokussierung}

Eine Distanzierung zum Geschehen lässt sich durch einen Perspektivenwechsel erzielen, der Blick auf die Situation kann gezoomt werden von der Einzelheit auf das Gesamte oder umgekehrt - s. u.: (1). Bei (2) schafft der Einsatz des nichtssagenden Unerwarteten eine Pause, verbunden mit einem Neustart. Bei (3) trägt die gedankliche Befragung imaginärer Dritter zur eigenen Beruhigung bei.

- (1) Gedanke: „Jetzt sitze ich hier Herrn Mustermann gegenüber, in seiner Wohnung, die Wohnung ist in der ... Straße, da wohnen mindestens 200 Familien, in der Stadt gibt es 200000 Einwohner, in dem Bundesland sind es schon 20 Millionen, in Deutschland 80, und wieviel Menschen leben in Europa?“

- (2) Frage: „Herr Mustermann, was haben Sie denn da für eine Krawatte um? Die sieht ja interessant aus. Wo haben Sie sie gekauft?“

- (3) Gedanke: „Was würde wohl jetzt mein erfahrener Kollege Klaus machen? Der würde erstmal cool bleiben und eine rauchen. Und dann schauen, was der Patient Interessantes zu berichten hat."

\section{Merke}

Anhaltendes Nachsinnen oder Grübeln über belastende Ereignisse gilt als Risiko für die Entwicklung einer posttraumatischen Belastungsstörung. Mithilfe psychotherapeutischer Techniken lässt es sich modifizieren. Resilienz im Umgang mit belastenden Ereignissen kann unter Supervision oder in einem Coaching trainiert werden.

\section{Spezielle Notfallpsychiatrie}

Alle Notfälle lassen sich aus psychiatrischer Sicht auf 5 Syndrome zurückführen. Diesen können sämtliche Erkrankungsbilder zugeordnet werden: 
- Tab. 3 Psychomotorische Erregungszustände.

\begin{tabular}{|c|c|}
\hline Parameter & Charakteristika \\
\hline Symptomatik & $\begin{array}{l}\text { " ziellose Steigerung von Antrieb und Psychomotorik } \\
\text { " affektive Enthemmung } \\
\text { - Gereiztheit, unvermittelte Gewaltausbrüche (Raptus) } \\
\text { - Kontrollverlust }\end{array}$ \\
\hline $\begin{array}{l}\text { mögliche Ursachen } \\
\text { und Risikofaktoren }\end{array}$ & $\begin{array}{l}\text { " unspezifisch } \\
\text { - vor allem Drogenintoxikationen (s. a. — Tab. 4) und komorbide Persönlichkeitsstörungen mit hohem } \\
\text { Aggressionspotenzial } \\
\text { - seltener Angst- und Panikstörungen, agitierte Depression, akute Exazerbationen einer schizophrenen } \\
\text { Erkrankung, Manie oder Demenz } \\
\text { - hirnorganisch, z. B. } \\
\text { - Schädel-Hirn-Trauma } \\
\text { - postiktaler Dämmerzustand } \\
\text { - infektiös } \\
\text { - Tumor } \\
\text { - organisch, z. B. Hyperthyreose }\end{array}$ \\
\hline $\begin{array}{l}\text { nichtpharmakologi- } \\
\text { sche Intervention }\end{array}$ & $\begin{array}{l}\text { - Prophylaxe, gefährliche Situationen rechtzeitig aus dem Verhalten und den Umständen erkennen und } \\
\text { vorbeugend Hilfe organisieren, für „sichere Räumlichkeiten“ sorgen, ggf. Polizei um Amtshilfe ersuchen, in } \\
\text { Klinik Werkschutz oder Wachdienst hinzuzuziehen } \\
\text { " „Talking down“, beruhigendes Zureden, Aufbau einer Vertrauensbasis, Einsatz von beschreibender Spra- } \\
\text { che zum Erläutern des weiteren Vorgehens } \\
\text { " „Time-out“, Verbringen in eine reizarme Umgebung, stationäre Aufnahme oder Umsetzen einer anti- } \\
\text { aggressiven Medikation (freiwillig oder zwangsweise) } \\
\text { " Fixierung, ggf. Zwangseinweisung (z. B. PsychKG) oder Überstellung an Polizei (Polizeigewahrsam) }\end{array}$ \\
\hline $\begin{array}{l}\text { pharmakologische } \\
\text { Intervention }\end{array}$ & $\begin{array}{l}\text { psychotische Erregung und Aggressivität bei Schizophrenie und manischen Syndromen } \\
\text { " Haloperidol } \\
\text { " Olanzapin } \\
\text { - Lorazepam } \\
\text { Erregung bei depressiven Syndromen } \\
\text { - Lorazepam } \\
\text { Erregung bei Angststörungen mit/ohne Panikattacken } \\
\text { - Lorazepam } \\
\text { Erregung bei symptomatischen Psychosen im Rahmen somatischer Erkrankungen (internistisch/neurolo- } \\
\text { gisch/Intoxikationen) } \\
\text { " kausale Pharmakotherapie } \\
\text { " ergänzend Haloperidol, Melperon } \\
\text { Erregung bei Drogenintoxikationen, Erregung bei pathologischem Alkoholrausch (massiver Erregungs- } \\
\text { zustand bei geringer Alkoholmenge<2\% mit gewalttätigem Verhalten, kompletter Amnesie für den Zustand } \\
\text { und Terminalschlaf, oft von kurzer Dauer) } \\
\text { - Haloperidol, Cave: Keine Benzodiazepine bei Alkoholintoxikation } \\
\text { Erregung bei Delir } \\
\text { - je nach Form } \\
\quad \text { - Haloperidol } \\
\text { - Clomethiazol } \\
\quad \text { - Benzodiazepine }\end{array}$ \\
\hline Besonderheiten & $\begin{array}{l}\text { Die Einnahme einer gemütlichen Sitzposition ist erregten oder gespannten Menschen nur begrenzt möglich. } \\
\text { Der Umgang kann ein „Management by walking around“ erfordern. Dabei ist immer auf das Bestehenbleiben } \\
\text { des Sicht-/Rufkontaktes zu weiterem Hilfspersonal zu achten. } \\
\text { Falls ein geordnetes Setting möglich ist, wird der Behandler direktiver vorgehen und in seiner Haltung be- } \\
\text { stimmter auftreten. Er wird Freiraum gewähren, ggf. aber auch Grenzen setzen müssen. Er sollte sich nicht } \\
\text { von dem Verhalten des Patienten provozieren lassen und gleichbleibend Ruhe ausstrahlen [6]. }\end{array}$ \\
\hline
\end{tabular}

- psychomotorische Erregungszustände (

- delirante Syndrome ( $\triangleright$ Tab. 5), diese können auch bei den Bewusstseinsstörungen subsumiert werden,

- akute Suizidalität ( $\vee$ Tab. 6),

- stuporöse Zustände ( $>$ Tab. 7),

- Störungen des Bewusstseins (๖ Tab. 8).

\section{Psychomotorische Erregungszustände}

Syndrom: Psychomotorische Erregungszustände

In ₹ Tab. 3 sind die für den Notarztdienst relevanten Charakteristika der psychomotorischen Erregungszustände zusammengefasst. 
FAZIT

Die Intervention zielt auf eine frühzeitige nicht pharmakologische Deeskalation. Falls erforderlich, kommen beruhigende, nach Möglichkeit nicht sedierende Medikamente zur Anwendung. Die Berücksichtigung der Wünsche des Patienten zur Medikation fördert den Beziehungsaufbau und stärkt gleichzeitig seinen Selbstwert.

Bei der Beziehungsgestaltung zu schizophrenen Patienten besteht die Herausforderung, den Patienten in seiner Persönlichkeit, d. h. auch in seinem paranoid-halluzinatorischen Erleben, zu akzeptieren, aber gleichzeitig Zweifel am realitätsverzerrten Erleben in Gang zu setzen, die Realitätsprüfung zu fördern und der Systematisierung des Wahns frühzeitig entgegenzuwirken. Die Gesprächsführung erfolgt entlang eines sogenannten sokratischen Dialogs, wobei durch geleitete, zielgenaue Fragen dem Betreffenden Lücken, Fehlschlüsse und Widersprüche in seinen pseudologischen Erklärungen aufgezeigt werden.

\section{FALLBEISPIEL}

\section{Gesprächsführung bei wahnhaftem Erleben}

„Herr Mustermann, Sie erwähnten, dass Ihre Schmerzen durch einen Alien im Bauch verursacht werden. "Herr Mustermann beschreibt die Beschwerden, die der Alien ihm zufügt, z. B. Völlegefühl, Aufstoßen und Darmkrämpfe. „Hhm, ich verstehe. Wie groß ist der Alien?" Herr Mustermann antwortet handballgroß. „Wie ein Handball? Wie ist er denn hineingekommen, Ihre Mundöffnung und Ihre Speiseröhre sind doch viel enger?"

Herr Mustermann führt aus, dass er ihn beim Essen eines Apfels verschluckt habe, als er noch kleiner gewesen sei. „Ah, Sie glauben, dass er gewachsen ist. Wie viel haben Sie denn in der letzten Zeit gegessen?“... „So wie sonst.“ ... „Dann kann er nicht gewachsen sein. Bei gleichmäßiger Essensmenge müssten Sie woanders abgenommen haben, wenn der Alien gewachsen ist. Haben Sie vielleicht ein altes Foto von sich auf dem Handy?“ ... „Das sieht unverändert aus. Herr Mustermann, ich glaube Ihnen gerne, dass Sie Leibbeschwerden haben, als ob Ihnen ein Alien im Bauch steckt. Sie sitzen mir ja auch ganz verkrampft gegenüber. Und ich glaube nicht, dass Sie schwindeln, so wie ich Sie jetzt hier erlebt habe. Was haben Sie denn in letzter Zeit gegessen?“...

„Da sind einige blähende Dinge darunter. Die könnten auch solche Beschwerden machen. “ Herr Mustermann äußert, dass er das nicht glaubt, zumal er auch ein Brennen im Oberbauch verspüre. „Okay, ja, da haben Sie recht. Das wird durch blähende Nahrung nicht hervorgerufen. Hatten Sie Stress in der letzten Zeit?“ Herr Mustermann beschreibt Stress am Arbeitsplatz mit einem neuen Vorarbeiter. „Na, vielleicht rührt es daher, dass aufgrund des Stresses Ihr Magen verstärkt Säure bildet. Die greift jetzt die Schleimhäute an und führt zu den Beschwerden. Vielleicht sollten wir versuchen, etwas gegen Ihren Stress zu machen?“
Merke

Falls eine Zwangsmaßnahme notwendig ist, sollte sie rasch unter Berücksichtigung der Würde des Betroffenen ohne weitere Demütigung, unter Ausschluss der Öffentlichkeit durchgeführt werden. Auch in kritischen Situationen unter Hektik und Gewalt sind ein respektvoller Umgang und eine höfliche - wenn auch bestimmte - Anrede möglich. Die Zwangsbehandlung sollte sich für den Patienten erkenntlich und nachvollziehbar in einen Gesamtbehandlungsplan einfügen.

Für die Basistherapie mit einem Antipsychotikum steht Haloperidol zur Verfügung, die Dosis beträgt 5-10 mg p.o. oder i.m., ggf. kann 1-2× nach 30 min wiederholt werden; eine Tagesdosis von $30 \mathrm{mg}$ sollte i.d.R. nicht überschritten werden, die maximale Dosis ist $50 \mathrm{mg} / \mathrm{d}$ (in seltenen Ausnahmefällen 100 mg p.o. pro Tag).

Alternativ kann Olanzapin verwendet werden, 10-20 mg p. o. oder $10 \mathrm{mg}$ i. m. (Cave: kein Lorazepam oder ein anderes Benzodiazepin bei i. m. Olanzapin), bis $30 \mathrm{mg} / \mathrm{d}$.

Steht die Sedierung im Vordergrund, ist Melperon 50100 mg p. o. oder i. m., bis maximal 200 mg/d, geeignet.

Eine zusätzliche Komedikation könnte Lorazepam sein, 12,5 mg p.o. (Expidet) oder 1-2 mg i.v. (langsam!)/i.m., ggf. nach 30 min wiederholen, bis maximal $10 \mathrm{mg} / \mathrm{d}$.

Clomethiazol (Distraneurin ${ }^{\circledR}$ ) wirkt sedierend, hypnotisch und antikonvulsiv, es ist für die stationäre Behandlung von akuten Entzugssymptomen, Prädelir und Delir zugelassen. Eine ambulante Verordnung ist nicht erlaubt! Wegen seiner kurzen Halbwertszeit von rund $4 \mathrm{~h}$ ist es gut steuerbar. Die Dosierung erfolgt flexibel an der Ausprägung der Entzugssymptomatik und des Sedierungsgrades orientiert. Da Clomethiazol atemdepressiv wirkt, sollte es nicht verwendet werden [6]

- bei einem Atem-/Blutalkohol >1,0\%,

- bei Benzodiazepin-Begleitmedikation,

- bei Hinweis auf allergische Reaktion in der Vorgeschichte (Nies- oder Hustenreiz?),

- bei respiratorischer Insuffizienz oder obstruktiven Lungenerkrankungen (COPD).

\section{Drogenabusus}

Den sogenannten pathognomischen Drogenblick [6] fasst $>$ Tab. 4 zusammen.

\section{Delirante Syndrome}

Die notfallmedizinisch relevanten Aspekte der deliranten Syndrome sind in $>$ Tab. 5 zusammengestellt.

\section{Akute Suizidalität}

Die Besonderheiten der akuten Suizidalität [6] im Rettungsdienst fasst $\vee$ Tab. 6 zusammen. 
- Tab. 4 Pathognomischer Drogenblick.

\begin{tabular}{|c|c|c|}
\hline Substanzen & Kennzeichen & \\
\hline \multirow{4}{*}{$\begin{array}{l}\text { Alkohol } \\
\text { Barbiturate } \\
\text { Benzodiazepine } \\
\text { Opiate }\end{array}$} & allgemeine Wirkung & $\begin{array}{l}\text { - beruhigend } \\
\text { - euphorisierend }\end{array}$ \\
\hline & bei Intoxikation & " zentralnervös dämpfend: Somnolenz bis Koma, Atemdepression \\
\hline & bei Entzug & $\begin{array}{l}\text { zentralnervös und vegetativ aktivierend: } \\
\text { - Angst, Unruhe } \\
\text { - Tremor, Schwitzen } \\
\text { - Diarrhö } \\
\text { - Blutdruck- und Pulsanstieg } \\
\text { - Glieder-, Muskelschmerzen }\end{array}$ \\
\hline & Besonderheiten & $\begin{array}{l}\text { - Opiatintoxikation } \\
\text { - Miosis } \\
\text { - Obstipation } \\
\text { - Opiatentzug } \\
\text { - Mydriasis } \\
\text { - lebhafte Darmgeräusche } \\
\text { - kein Delir } \\
\text { - zeitliche Entwicklung in Abhängigkeit der Halbwertszeit: } \\
\quad \text { Heroin 6-8 h, Methadon 24-36 h nach letzter Einnahme } \\
\text { " „trockenes Alkoholdelir“ mit geringer/fehlender vegetativer Sympto- } \\
\text { matik bei Polyneuropathie } \\
\text { - Cave: Krampfanfälle im Entzug }\end{array}$ \\
\hline \multirow[t]{4}{*}{$\begin{array}{l}\text { Cannabis (Haschisch, } \\
\text { Marihuana) }\end{array}$} & allgemeine Wirkung & $\begin{array}{l}\text { - beruhigend } \\
\text { - halluzinogen }\end{array}$ \\
\hline & bei Intoxikation & $\begin{array}{l}\text { - Übelkeit, Erbrechen } \\
\text { - Hypothermie } \\
\text { - Wahnvorstellungen, Halluzinationen }\end{array}$ \\
\hline & bei Entzug & $\begin{array}{l}\text { vor allem psychische Beschwerden: } \\
\text { - depressive Verstimmung } \\
\text { " Reizbarkeit, Ängstlichkeit }\end{array}$ \\
\hline & Besonderheiten & " amotivationales Syndrom \\
\hline \multirow{4}{*}{$\begin{array}{l}\text { Amphetamine (Am- } \\
\text { phetamin, Metamphe- } \\
\text { tamin [u. a. Speed, } \\
\text { Crystal]) } \\
\text { MDA [Eve] } \\
\text { MDMA [Ecstasy] } \\
\text { Kokain (u. a. Crack) }\end{array}$} & allgemeine Wirkung & $\begin{array}{l}\text { - anregend } \\
\text { " euphorisierend }\end{array}$ \\
\hline & bei Intoxikation & $\begin{array}{l}\text { ausgeprägte sympathoadrenerge zentrale und vegetative Effekte: } \\
\text { - Unruhe, Agitation } \\
\text { " Reizbarkeit, Aggressivität } \\
\text { " psychotisches Erleben, Halluzinationen } \\
\text { - zerebrale Krampfanfälle, Hyperreflexie } \\
\text { - Vasokonstriktionen unterschiedlicher Stromgebiete mit erhöhtem In- } \\
\text { farktrisiko (z. B. Hirn-, Herz-, Nieren-, Darminfarkt) } \\
\text { - schwere Gerinnungsstörungen bis zur disseminierten intravasalen } \\
\text { Gerinnung } \\
\text { - Hypertonie } \\
\text { - Tachykardie } \\
\text { - Hyperthermie, Fieber }\end{array}$ \\
\hline & bei Entzug & $\begin{array}{l}\text { untypischer, z. T. wochenlanger Verlauf: } \\
\text { - ausgeprägte Depressionen, Suizidalität } \\
\text { - Angstzustände } \\
\text { - Antriebslosigkeit, Erschöpfung } \\
\text { - Heißhunger mit Hyperphagie }\end{array}$ \\
\hline & Besonderheiten & $\begin{array}{l}\text { - MDMA-Intoxikation klinisch abgeschwächter, aber mit Todesfällen } \\
\text { - Kokainschock } \\
\text { - unmittelbar nach Einnahme } \\
\text { - akut lebensbedrohlich }\end{array}$ \\
\hline
\end{tabular}


- Tab. 4 Fortsetzung

\begin{tabular}{|c|c|c|}
\hline Substanzen & Kennzeichen & \\
\hline \multirow{4}{*}{$\begin{array}{l}\text { Psychotomimetika } \\
\text { (heterogene Gruppe, } \\
\text { u. a. LSD, Meskalin, Psi- } \\
\text { locybin, Phencyclidin } \\
\text { [„Angel Dust“]) }\end{array}$} & allgemeine Wirkung & $\begin{array}{l}\text { " anregend, halluzinogen } \\
\text { - bereits in geringen Mengen rauschartige Bewusstseinsveränderungen } \\
\text { und halluzinatorisches Erleben }\end{array}$ \\
\hline & bei Intoxikation & $\begin{array}{l}\text { vor allem zentralserotonerg wirksam, mit peripheren Intoxikations- } \\
\text { erscheinungen erst bei sehr hohen Dosen: } \\
\text { " psychotische Symptomatik mit akustischen und optischen Halluzina- } \\
\text { tionen, Wahnvorstellungen, Angst- und Erregungszuständen } \\
\text { " im Verlauf Vigilanzstörungen bis zum Koma, Mydriasis, Nystagmus, } \\
\text { Hyperreflexie, zerebrale Krampfanfälle, körperlich Übelkeit, Erbre- } \\
\text { chen, Fieber, Hypertension, Tachykardie }\end{array}$ \\
\hline & bei Entzug & - kein spezifisches Entzugssyndrom \\
\hline & Besonderheiten & $\begin{array}{l}\text { - schnelle Toleranzentwicklung mit Rückbildung nach Absetzen } \\
\text { " physische und psychische Abhängigkeiten selten } \\
\text { - Horrortrips mit eigen- und fremdaggressivem Verhalten } \\
\text { " Flashback-Psychosen noch Monate verzögert, evtl. dosisunabhängig }\end{array}$ \\
\hline
\end{tabular}

LSD = Lysergsäurediethylamid; MDA = Methylendioxyamphetamin; MDMA =3,4-Methylendioxymethamphetamin (Ecstasy)

- Tab. 5 Delirante Syndrome.

\begin{tabular}{|c|c|}
\hline Parameter & Charakteristika \\
\hline Symptomatik & $\begin{array}{l}\text { - Störungen von Bewusstsein (Klarheit } \downarrow \text { ) und Aufmerksamkeit } \\
\text { - Desorientiertheit } \\
\text { - Wahrnehmungsstörungen (optische Halluzinationen, illusionäre Verkennungen) } \\
\text { - erhöhte Suggestibilität } \\
\text { - psychomotorische Unruhe und Erregung } \\
\text { - fokal-neurologische Symptome (Ataxie, Dysarthrie, Tremor, Nesteln) } \\
\text { - vegetative Symptome (Hyperhidrosis, Tachykardie, Hypertonus, Diarrhö, Übelkeit, Erbrechen) }\end{array}$ \\
\hline $\begin{array}{l}\text { mögliche Ursachen und } \\
\text { Risikofaktoren }\end{array}$ & 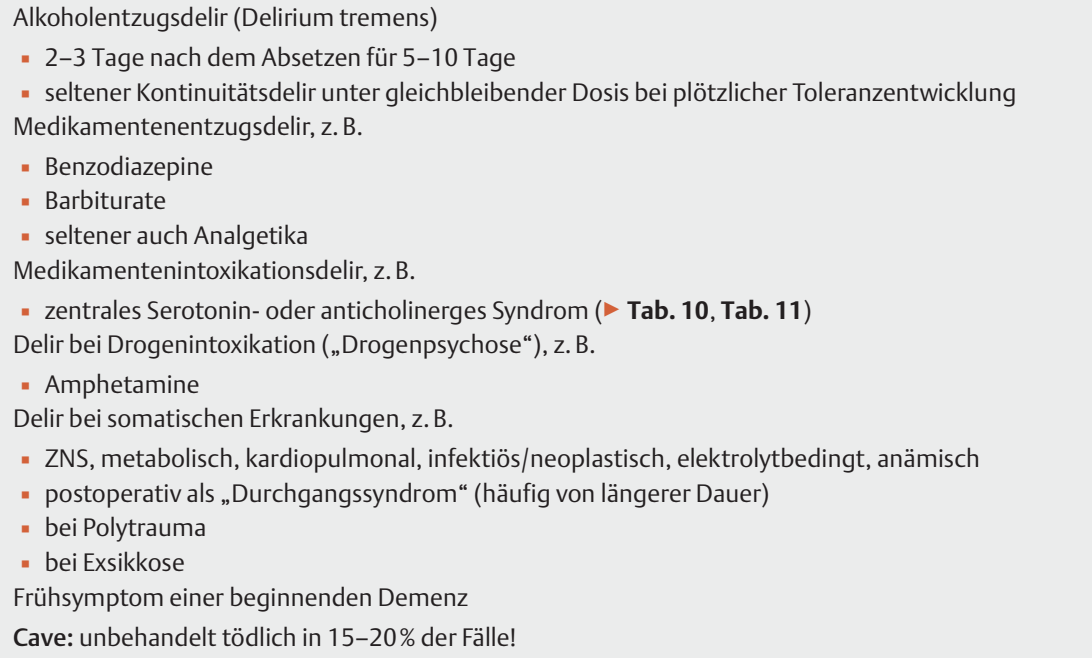 \\
\hline $\begin{array}{l}\text { nichtpharmakologische } \\
\text { Intervention }\end{array}$ & $\begin{array}{l}\text { - Reorientierungshilfen, Bezugspersonen, Angehörige } \\
\text { - Ausgleich sensorischer Defizite, geistige Betätigung } \\
\text { - Verringerung der Polypharmazie } \\
\text { - Beseitigung möglicher Auslöser (Infekte, Schmerzen, Schlafstörungen, Dehydratation/Elektrolyt- } \\
\text { dysbalancen, Konstipation/Urinretention, Malnutrition, Hypoxie und Immobilität) }\end{array}$ \\
\hline
\end{tabular}


Tab. 5 Fortsetzung

\begin{tabular}{|c|c|}
\hline Parameter & Charakteristika \\
\hline $\begin{array}{l}\text { pharmakologische } \\
\text { Intervention }\end{array}$ & $\begin{array}{l}\text { Basistherapie mit Antipsychotikum } \\
\text { - Haloperidol 1-2 mg p. o. oder i. m., alle 2-4 h, Titration nach Schweregrad, geringere Dosis bei } \\
\text { älteren Patienten } \\
\text { - Cave: Bei intravenöser Applikation kontinuierliches EKG-Monitoring wegen QT-Verlängerungen } \\
\text { und Torsades de Pointes } \\
\text { zusätzlich Clomethiazol } \\
\text { - } 2 \text { Kps. }(10 \mathrm{ml}) \text { alle } 1-2 \mathrm{~h} \text {, maximal } 24 \mathrm{Kps} . / \mathrm{d} \text {, } \\
\text { - Clomethiazol ist die Basistherapie des Alkoholentzugsdelirs. } \\
\text { - Cave: Gefahr einer Atem- und Kreislaufdepression, bronchiale Hypersekretion } \\
\text { alternativ Benzodiazepine } \\
\text { - z. B. Lorazepam 0,5-2 mg p. o. oder i.v. 2-4-stündlich, } \leq 7,5 \mathrm{mg} / \mathrm{d}\end{array}$ \\
\hline Besonderheiten & - trockenes Delir mit fehlender vegetativer Symptomatik \\
\hline
\end{tabular}

D Tab. 6 Akute Suizidalität.

\begin{tabular}{|c|c|}
\hline Parameter & Charakteristika \\
\hline Symptomatik & $\begin{array}{l}\text { ursachenabhängig unterschiedlich } \\
\text { anhedon-hoffnungsloser Typus } \\
\text { " Hoffnungslosigkeit, fehlende Zukunftsbezüge } \\
\text { - Gedankeneinengung, situative Einengung } \\
\text { " soziale Isolierung } \\
\text { " Aggressionsstau mit Wendung der Aggression gegen das eigene Ich } \\
\text { ängstlich agitierter Typus } \\
\text { " zunächst Aggressionshemmung nach außen, mit zunehmender Agitiertheit auch autoaggressive } \\
\text { Tendenzen } \\
\text { impulsiv-aggressiver Typus } \\
\text { Cave: Ausbrüche von bedrohlichem und gewalttätigem Verhalten }\end{array}$ \\
\hline $\begin{array}{l}\text { mögliche Ursachen und } \\
\text { Risikofaktoren }\end{array}$ & $\begin{array}{l}\text { Depression } \\
\text { " gedrückte Stimmung } \\
\text { - Antriebsminderung } \\
\text { - Verlust von Freude und Interesse } \\
\text { - Tagesschwankungen } \\
\text { " synthymer Wahn } \\
\text { Schizophrenie } \\
\text { - imperative (auffordernde) Stimmen, sich umzubringen } \\
\text { Persönlichkeitsstörung (vor allem emotional-instabil, narzisstisch) } \\
\text { " selbstverletzendes Verhalten und/oder Kränkungserleben } \\
\text { " psychosoziale Belastungen in der Vorgeschichte } \\
\text { akute Belastungsreaktionen } \\
\text { somatische Erkrankungen, vor allem chronische Schmerzen, Tumor } \\
\text { Risikofaktoren: } \\
\text { - konkrete Pläne, Vorbereitungen } \\
\text { - Suizidversuche in der Vorgeschichte } \\
\text { " Suizide in der Familie } \\
\text { - ältere alleinlebende Männer }\end{array}$ \\
\hline $\begin{array}{l}\text { nichtpharmakologische } \\
\text { Intervention }\end{array}$ & $\begin{array}{l}\text { " menschliche Gesellschaft, Kommunikationsangebot } \\
\text { " verständnisvolles Gespräch zu den Ursachen, dabei ggf. Ermöglichung einer Aggressionsabfuhr, } \\
\text { d. h. die Wendung der Aggression gegen die eigene Person aufheben bzw. umleiten } \\
\text { " Aufbau von Zukunftsbezügen } \\
\text { " Vermittlung in eine weitergehende Behandlung, z. B. stationäre Aufnahme } \\
\text { " ggf. auch Zwangseinweisung }\end{array}$ \\
\hline $\begin{array}{l}\text { pharmakologische } \\
\text { Intervention }\end{array}$ & $\begin{array}{l}\text { syndrom- bzw. störungsspezifisch, z. B. } \\
\text { = sedierend } \\
\text { " anxiolytisch } \\
\text { = bei begleitender psychotischer Erregung antipsychotisch } \\
\text { Die Einleitung einer antidepressiven Basistherapie ist durch den Notarzt nicht erforderlich. }\end{array}$ \\
\hline
\end{tabular}


- Tab. 6 Fortsetzung

\section{Parameter}

Besonderheiten

\section{Charakteristika}

- Bagatellisierende Aussagen „Alles wird gut“ oder „Deswegen bringt man sich doch nicht um“ sind destabilisierend.

- Die suizidale Problematik sollte nicht über den Patienten hinweg mit den Angehörigen erörtert werden.

- Die Exploration einer möglichen Hoffnungslosigkeit und/oder fehlender Zukunftsbezüge, z. B. Termine, ist geeignet, eine akute Suizidalität abzuschätzen.

- Da sich suizidale Patienten häufig auf die direkte Frage nach Suizidalität vorbereitet haben und sie mühelos verneinen können, empfiehlt es sich mitunter, eine paradoxe Intervention vorzunehmen. Die fehlende oder verzögerte Antwort auf die Frage: „Warum wollen Sie leben?“ sollte den Untersucher bewegen, von einer akuten Suizidalität auszugehen.

- Ein Non-Suizid-Pakt, sich von dem Betroffenen versprechen zu lassen, sich nichts anzutun, sollte bei fehlender therapeutischer Beziehung nicht geschlossen werden.

\ Tab. 7 Stuporöse Zustände.

\begin{tabular}{|c|c|}
\hline Parameter & Charakteristika \\
\hline Symptomatik & $\begin{array}{l}\text { - abnormer Zustand psychomotorischer Hemmung } \\
\text { - eingeschränkte bzw. aufgehobene Reaktivität auf Umweltreize } \\
\text { - voll erhaltenes Wachbewusstsein } \\
\text { - ursachenabhängig Besonderheiten im klinischen Bild }\end{array}$ \\
\hline $\begin{array}{l}\text { mögliche Ursachen } \\
\text { und Risikofaktoren }\end{array}$ & 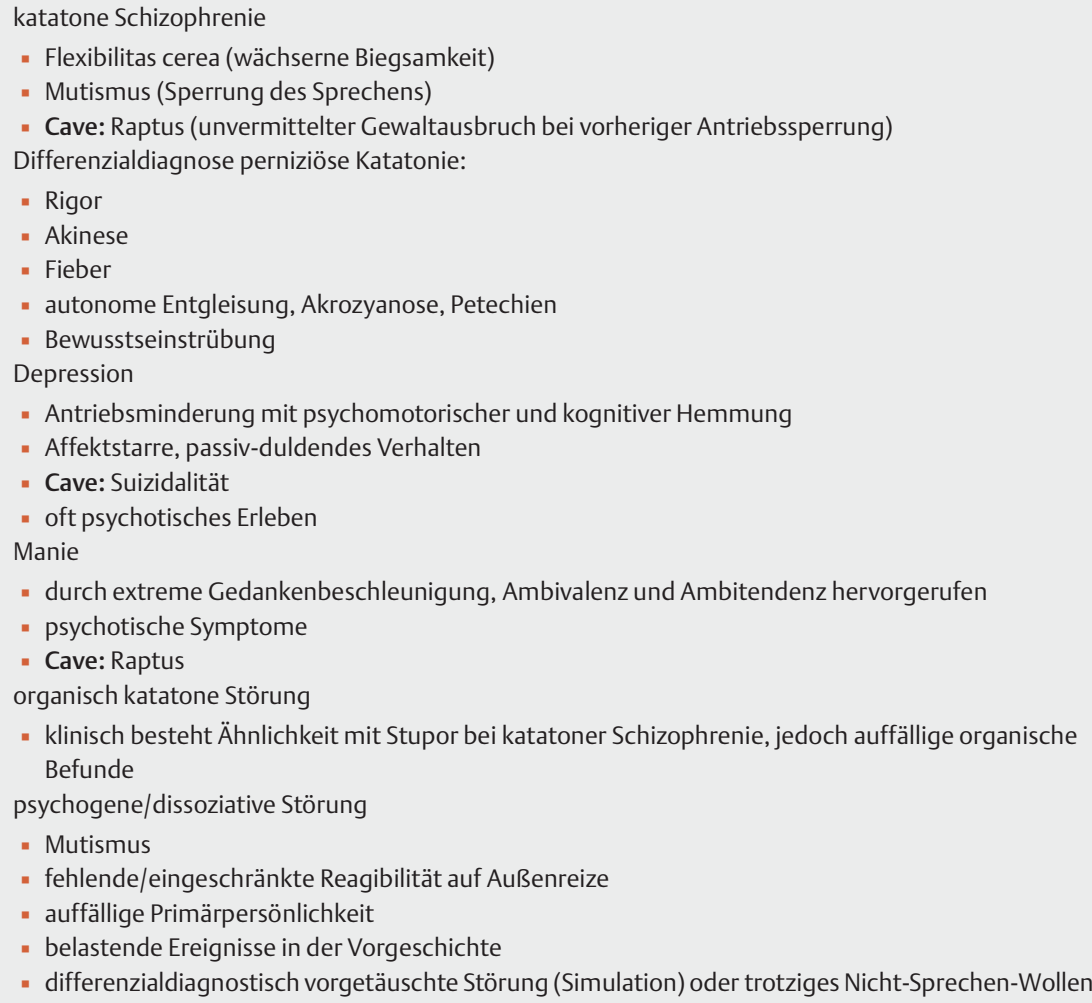 \\
\hline $\begin{array}{l}\text { nichtpharmakologische } \\
\text { Intervention }\end{array}$ & $\begin{array}{l}\text { - Reizabschirmung } \\
\text { - beruhigendes Gespräch bei psychogener Genese }\end{array}$ \\
\hline $\begin{array}{l}\text { pharmakologische } \\
\text { Intervention }\end{array}$ & $\begin{array}{l}\text { - bei Stupor unbekannter Genese initial Lorazepam 1-2,5 mg p. o. (Expidet) oder i.v. } \\
\text { - bei fehlender Wirkung nach Ausschluss eines malignen neuroleptischen Syndroms ( } \text { Tab. 9) } \\
\text { Haloperidol 5-10 mg p. o. oder i. m. }\end{array}$ \\
\hline Besonderheiten & $\begin{array}{l}\text { - Wenn i.v. Gabe von Haloperidol erforderlich ist, sollte ein EKG-Monitoring gewährleistet sein. } \\
\text { - Bei perniziöser Katatonie ist zusätzlich für Kühlung und Volumengabe zu sorgen. } \\
\text { - Nach Ausschluss einer Enzephalitis kann eine Elektrokrampftherapie indiziert sein. }\end{array}$ \\
\hline
\end{tabular}


- Tab. 8 Bewusstseinsstörungen.

\begin{tabular}{|c|c|}
\hline Parameter & Charakteristika \\
\hline $\begin{array}{l}\text { Sympto- } \\
\text { matik }\end{array}$ & $\begin{array}{l}\text { quantitativ } \\
\text { " physiologisch: Schlaf } \\
\text { " pathologisch: Somnolenz } \rightarrow \text { Sopor } \rightarrow \\
\text { Koma } \\
\text { qualitativ } \\
\text { " Einengung } \\
\text { " Verschiebung } \\
\text { " Erweiterung }\end{array}$ \\
\hline $\begin{array}{l}\text { mögliche } \\
\text { Ursachen } \\
\text { und Risiko- } \\
\text { faktoren }\end{array}$ & $\begin{array}{l}\text { quantitativ } \\
\text { - Intoxikation mit Sedativa und/oder } \\
\text { Alkohol } \\
\text { " metabolische Entgleisungen } \\
\text { " zerebrovaskuläres Ereignis } \\
\text { - Infektion des ZNS } \\
\text { " Schädel-Hirn-Trauma } \\
\text { qualitativ } \\
\text { - Delir } \\
\text { - organisch dissoziative Störung (Däm- } \\
\text { merzustand) bei Epilepsie, iktal und } \\
\text { postiktal } \\
\text { - bei pathologischem Alkoholrausch, } \\
\text { Drogenabusus } \\
\text { - Schädel-Hirn-Trauma oder entzündli- } \\
\text { chem Prozess }\end{array}$ \\
\hline $\begin{array}{l}\text { pharma- } \\
\text { kologische } \\
\text { Interven- } \\
\text { tion, Beson- } \\
\text { derheiten }\end{array}$ & $\begin{array}{l}\text { quantitativ } \\
\text { " intensivmedizinischer Notfall } \\
\text { qualitativ } \\
\text { " ursachenspezifisch } \\
\text { " nach Ausschluss Intoxikation } \\
\text { - bei epileptischer Genese Lorazepam } \\
0,5-2 \mathrm{mg} \text { i. v. } \\
\text { - bei pathologischem Alkoholrausch } \\
\text { Haloperidol 5-10 mg p. o. oder i. m. }\end{array}$ \\
\hline
\end{tabular}

Cave

Bei entsprechendem Verdacht ist eine mögliche Suizidalität immer zu explorieren. Je konkreter die Pläne und die Vorbereitungen sind, desto größer ist das Suizidrisiko.

\section{FALLBEISPIEL}

Umsetzung einer stationären Aufnahme bei depressiver Entscheidungsschwäche (Ambivalenz) durch Betonung der Wahlfreiheit

„Herr Mustermann, ich habe einen Rettungswagen gerufen, der wird Sie in die psychiatrische Klinik fahren. Möchten Sie erst mit Ihrer Frau telefonieren oder gleich losfahren?"

\section{Stuporöse Zustände}

Rettungsdienstliche Aspekte stuporöser Zustände sind durch einige Besonderheiten gekennzeichnet, die in - Tab. 7 zusammengestellt sind.
- Tab. 9 Malignes neuroleptisches Syndrom.

\begin{tabular}{|l|l|}
\hline Parameter & Charakteristika \\
\hline Definition & $\begin{array}{l}\text { sehr seltene Nebenwirkung einer Neuro- } \\
\text { leptikatherapie }\end{array}$ \\
\hline Medika- & klassische hochpotente Neuroleptika, \\
mente & auch Atypika \\
\hline Dosis & $\begin{array}{l}\text { vorwiegend hohe Dosen, auch normale } \\
\text { Dosen }\end{array}$ \\
\hline Beginn & innerhalb von 2 Wochen nach Anfang \\
\hline einer Neuroleptikatherapie
\end{tabular}

\section{Störungen des Bewusstseins}

Störungen des Bewusstseins sind ein bedeutender Gesichtspunkt im notärztlichen Setting. > Tab. 8 fasst die Besonderheiten zusammen.

Psychopharmaka können selbst Ursache psychiatrischer Notfälle sein. Der Verdacht auf ein malignes neuroleptisches Syndrom, ein zentrales anticholinerges Syndrom 
- Tab. 10 Zentrales anticholinerges Syndrom.

\begin{tabular}{|c|c|}
\hline Parameter & Charakteristika \\
\hline Definition & $\begin{array}{l}\text { Nebenwirkung anticholinerg wirksamer } \\
\text { Pharmaka }\end{array}$ \\
\hline $\begin{array}{l}\text { Medika- } \\
\text { mente }\end{array}$ & $\begin{array}{l}\text { " z. B. Clozapin } \\
\text { " trizyklische Neuroleptika (TZ-NL) } \\
\text { " trizyklische Antidepressiva (TZA) }\end{array}$ \\
\hline Dosis & $\begin{array}{l}\text { Überdosierung bzw. Intoxikation, auch } \\
\text { normale Dosen bei Slow-Metabolizer }\end{array}$ \\
\hline Verlauf & potenziell lebensbedrohlich \\
\hline $\begin{array}{l}\text { Sympto- } \\
\text { matik }\end{array}$ & $\begin{array}{l}\text { periphere anticholinerge Symptome } \\
\text { " trockene Haut, Hyperthermie } \\
\text { - Harnverhalt, Obstipation } \\
\text { " tachykarde Herzrhythmusstörungen } \\
\text { " Mydriasis } \\
\text { zentrale Symptome } \\
\text { - Delir } \\
\text { " Agitiertheit } \\
\text { " auch sedativ mit Somnolenz/Koma }\end{array}$ \\
\hline $\begin{array}{l}\text { Komplika- } \\
\text { tionen }\end{array}$ & $\begin{array}{l}\text { - Herzrhythmusstörungen } \\
\text { " paralytischer lleus } \\
\text { - Elektrolytentgleisungen } \\
\text { - epileptische Anfälle } \\
\text { - Koma }\end{array}$ \\
\hline \multirow[t]{2}{*}{ Therapie } & $\begin{array}{l}\text { Absetzen der Medikation, } \\
\text { ggf. Sedierung mit Benzodiazepine/Anti- } \\
\text { psychotika }\end{array}$ \\
\hline & $\begin{array}{l}\text { Physostigmin (Anticholium }{ }^{\circledR} \text { Injektions- } \\
\text { Isg.) 2-4 mg i. m. oder langsam i.v. }\end{array}$ \\
\hline
\end{tabular}

oder ein zentrales Serotoninsyndrom erfordert die sofortige Einweisung in eine Klinik. Eine Übersicht über das maligne neuroleptische Syndrom, das zentrale anticholinerge Syndrom und das zentrale Serotoninsyndrom geben $>$ Tab. 9, 10 und Tab. 11).

\section{KERNAUSSAGEN}

- Psychiatrische Notfälle gehören zu den häufigsten Einsatzursachen für Notärzte.

- Sämtliche psychiatrischen Notfälle lassen sich den 5 Syndromen psychomotorische Erregungszustände, Delir, akute Suizidalität, stuporöse Zustände,

Störungen des Bewusstseins zuordnen.

- Das Setting ist an die Erfordernisse aus der Notsituation und dem Störungsbild des Hilfesuchenden anzupassen. Dabei ist auf die persönliche Sicherheit zu achten.

- Das Vorgehen erfolgt störungsspezifisch.

- Das kommunikative Geschick des Ersthelfers ist das wichtigste Interventionsinstrument.

- Die Notfallmedikation lässt sich auf wenige Psychopharmaka beschränken.
- Tab. 11 Zentrales Serotoninsyndrom.

\begin{tabular}{|c|c|}
\hline Parameter & Charakteristika \\
\hline Definition & $\begin{array}{l}\text { seltene Neben-/Wechselwirkung seroto- } \\
\text { nerg wirksamer Pharmaka }\end{array}$ \\
\hline $\begin{array}{l}\text { Medika- } \\
\text { mente }\end{array}$ & $\begin{array}{l}\text { " selektive Serotoninwiederaufnahme- } \\
\text { hemmer (SSRI) } \\
\text { - selektive Serotonin- und Noradrena- } \\
\text { linwiederaufnahmehemmer (SSNRI) } \\
\text { - trizyklische Antidepressiva (TZA) } \\
\text { - Monoaminoxidashemmer (MAOH) } \\
\text { - Serotonin-(5-HT-)Agonisten } \\
\text { - Kokain } \\
\text { - Amphetamine } \\
\text { - Lithium }\end{array}$ \\
\hline Beginn & $\begin{array}{l}\text { innerhalb der ersten } 24 \mathrm{~h} \text { nach Applika- } \\
\text { tion }\end{array}$ \\
\hline Verlauf & potenziell lebensbedrohlich \\
\hline $\begin{array}{l}\text { Sympto- } \\
\text { matik }\end{array}$ & $\begin{array}{l}\text { Trias } \\
\text { " Fieber } \\
\text { " neuromuskuläre Symptome (Hyper- } \\
\text { rigidität, Hyperreflexie, Myokloni, } \\
\text { Tremor) } \\
\text { " psychopathologische Auffälligkeiten } \\
\text { (Delir, Erregungszustände) }\end{array}$ \\
\hline $\begin{array}{l}\text { Komplika- } \\
\text { tionen }\end{array}$ & $\begin{array}{l}\text { - epileptische Anfälle } \\
\text { " Herzrhythmusstörungen } \\
\text { " Koma } \\
\text { " Verbrauchskoagulopathie } \\
\text { - Multiorganversagen }\end{array}$ \\
\hline \multirow[t]{3}{*}{ Therapie } & $\begin{array}{l}\text { Absetzen der Medikation (in 90\% der } \\
\text { Fälle ausreichend), }\end{array}$ \\
\hline & $\begin{array}{l}\text { Kühlung, Volumensubstitution, ggf. } \\
\text { Sedierung }\end{array}$ \\
\hline & $\begin{array}{l}\text { Cyproheptadin }\left(\text { Peritol }^{\circledR}\right) 4-8 \mathrm{mg} \text { initial } \\
\text { p. o. bis } 0,5 \mathrm{mg} / \mathrm{kgKG} / \mathrm{d}\end{array}$ \\
\hline
\end{tabular}

Interessenkonflikt

Der Autor gibt an, dass keine Interessenkonflikte vorliegen.

Über den Autor

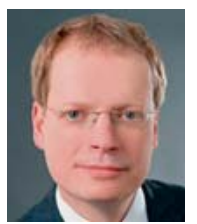

\section{Wolfgang Jordan}

Prof. Dr. med., MBA, MIM. Stellv. Ärztlicher Direktor der Klinikum Magdeburg gGmbH, Chefarzt der Klinik für Psychiatrie und Psychotherapie. Facharzt für Psychiatrie und Psychotherapie, Schlafmedizin, Suchtmedizin, VideoInterventions-Therapie. Studium der Managementwissenschaften Universität Salzburg, außerplanmäßige Professur Universität Göttingen. Publikationsschwerpunkte: Notfallpsychiatrie, Schlafmedizin, oxidativer Stress, MutterKind-Behandlung, Management, Ethik. 
Korrespondenzadresse

Prof. Dr. med. Wolfgang Jordan, MBA, MIM

Klinik für Psychiatrie und Psychotherapie

Klinikum Magdeburg gGmbH

Birkenallee 34

39130 Magdeburg

wolfgang.jordan@klinikum-magdeburg.de

Wissenschaftlich verantwortlich

gemäß Zertifizierungsbestimmungen

Wissenschaftlich verantwortlich gemäß Zertifizierungsbestimmungen für diesen Beitrag ist Prof. Dr. med. W. Jordan, MBA, MIM, Magdeburg.

\section{Literatur}

[1] Flüchter $P$, Müller $V$, Bischof $F$ et al. Notarztschulung über psychiatrische Notfälle: Evaluation eines interaktiven Fortbildungsprogramms. Psychiat Prax 2017; 44: 105-107
[2] Pajonk FG. Zur Situation der Notfall- und Akutpsychiatrie in Deutschland. Nervenarzt 2015; 86: 1081-1090

[3] Pajonk FG, Lubda J, Sittinger $\mathrm{H}$ et al. Psychiatrische Notfälle aus der Sicht von Notärzten - eine Reevaluation nach 7 Jahren. Anaesthesist 2004; 53: 709-716

[4] Pajonk FG, Gärtner U, Sittinger $\mathrm{H}$ et al. Psychiatrische Notfälle aus der Sicht von Rettungsdienstmitarbeitern. Notfall Rettungsmed 2004; 7: 161-167

[5] Wild J, Smith KV, Thompson E et al. A prospective study of pretrauma risk factors for post-traumatic stress disorder and depression. Psychol Med 2016; 46: 2571-2582

[6] Jordan W, Heinemann A, Marx A, Hrsg. Notfallpsychiatrie und psychotherapeutische Krisenintervention. Stuttgart, New York: Thieme; 2016

Bibliografie

DOI https://doi.org/10.1055/s-0043-117554

Notarzt 2017; 33: 224-241

(c) Georg Thieme Verlag KG Stuttgart · New York ISSN 0177-2309 
Punkte sammeln auf CME.thieme.de

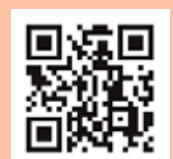

Diese Fortbildungseinheit ist 12 Monate online für die Teilnahme verfügbar.

Sollten Sie Fragen zur Online-Teilnahme haben, finden Sie unter cme.thieme.de/hilfe eine ausführliche Anleitung. Wir wünschen viel Erfolg beim Beantworten der Fragen!

Unter eref/thieme.de/ZZX9ZWC oder über den QR-Code kommen Sie direkt zum Artikel zur Eingabe der Antworten.

VNR 2760512017152373363

\section{Frage 1}

Eine der folgenden Aussagen ist falsch. Welche?

A Mit einem Anteil von $50 \%$ sind psychiatrische Notfälle die häufigste Einsatzursache für Notärzte.

B Psychiatrische Notfälle werden von dem Tätigen im Rettungsdienst als besonders belastend empfunden.

C Psychiatrische Notfälle können bei Rettungssanitätern posttraumatische Belastungsstörungen oder Depressionen auslösen.

D Anhaltendes Nachsinnen oder Grübeln über belastende Ereignisse gilt als Risiko für die Entwicklung einer posttraumatischen Belastungsstörung.

E Psychiatrische Notfälle können auch bei Patienten mit somatischer Erkrankung auftreten.

\section{Frage 2}

Welche Aussage ist richtig?

A Ideenflucht und Parathymie weisen auf eine schizophrene Erkrankung hin.

B Affektinkontinenz, Weitschweifigkeit und Perseverationen sind hinweisend auf hirnorganische Erkrankungen.

C Patienten mit einer wahnhaften Depression leiden zumeist unter Verfolgungswahn.

D Optische Halluzinationen sind ein typischer Befund der Schizophrenie.

E Ichstörungen kommen nur bei an Schizophrenie Erkrankten vor.

\section{Frage 3}

Welche Aussage trifft zu?

A Primäres Behandlungsziel bei psychiatrischen Notfällen ist Sedierung.

B Primäres Behandlungsziel bei psychiatrischen Notfällen ist Beruhigung.

C In der Notfallpsychopharmakotherapie sollten trizyklische Antipsychotika in Kombination mit sedierenden Benzodiazepinen zur Anwendung kommen.

D Diazepam ist Lorazepam vorzuziehen, da es einen schnelleren Wirkungseintritt hat und wegen fehlender aktiver Metaboliten besser steuerbar ist.

E Die intramuskuläre Gabe von Olanzapin eignet sich gut zur Kombinationsbehandlung mit Benzodiazepinen.

\section{Frage 4}

Eine der folgenden Aussagen zum Entzug ist nicht zutreffend. Welche?

A Ein Alkoholentzugsdelir (Delirium tremens) tritt 2-3 Tage nach dem Absetzen für eine Dauer von 5-10 Tagen auf.

B Ein Alkoholentzugsdelir kann auch bei kontinuierlicher Trinkmenge auftreten.

C Ein Medikamentenentzugsdelir findet sich vor allem bei Benzodiazepinen und Barbituraten.

D Ein Medikamentenintoxikationsdelir kann bei dem zentralen Serotonin- oder anticholinergen Syndrom bestehen.

E Der kalte Entzug von Opiaten geht mit einem erhöhten Risiko, ein Delir zu entwickeln, einher.

\section{Frage 5}

Welche Aussage ist nicht korrekt?

A Psychiatrisch-psychotherapeutische Notfälle können mit Selbst- und/oder Fremdgefährdung einhergehen.

B Bei den vorgesehenen Gesprächspartnern ist eine ausreichende Motivation, Belastbarkeit und Kommunikationsfähigkeit zu prüfen.

C Bei Menschen in psychischen Krisen sind oft eine Einengung der Wahrnehmung auf die Themen Kränkung und Verlust, Schwarz-Weiß-Denken sowie eine interne Kommunikationsstörung mit nachfolgender Missdeutung der beteiligten Kommunikationspartner zu finden.

D Die Örtlichkeit der Krisenintervention ist entsprechend der vorbekannten, ersichtlichen oder zu erwartenden Beeinträchtigung auszuwählen und ggf. an die Erfordernisse anzupassen. Dabei ist auf die persönliche Sicherheit zu achten.

E Eine frühzeitige Deutung und Interpretation verbessert den Beziehungsaufbau zum Patienten.

\section{- Weitere Fragen auf der folgenden Seite...}




\title{
Punkte sammeln auf CMIE, thieme.de
}

\author{
Fortsetzung ...
}

\section{Frage 6}

Welche Aussage zu psychomotorischen Erregungszuständen stimmt nicht?

A Psychomotorische Erregungszustände werden ausschließlich pharmakologisch behandelt.

B Psychomotorische Erregungszustände können auch somatisch begründete Ursachen haben.

C Psychomotorische Erregungszustände können eine Zwangseinweisung (z.B. PsychKG) oder eine Überstellung in den Polizeigewahrsam erfordern.

D Psychomotorische Erregungszustände können ein „Management by walking around" erfordern.

E Psychomotorische Erregungszustände können medikamentös zumeist gut mit Haloperidol und Lorazepam behandelt werden.

\section{Frage 7}

Welche Aussage gilt?

A Bei akuter Suizidalität sollte noch in der Notfallsituation ein Non-Suizid-Pakt mit dem Betreffenden geschlossen werden.

B Bagatellisierende Aussagen wie „Alles wird gut“ oder „Deswegen bringt man sich doch nicht um “ destabilisieren den Betreffenden und sollten unterbleiben.

C Akute Suizidalität gibt es nur bei psychiatrischen Patienten.

D Akute Suizidalität lässt sich am besten durch die Befragung der Angehörigen ohne belastende Einbindung des Patienten in seinem Beisein klären.

E Akute Suizidalität ist durch Zwangsmaßnahmen gut zu behandeln.

\section{Frage 8}

Eine der folgenden Aussagen zur Suizidalität trifft nicht zu.

Welche?

A Akute Suizidalität erfordert unmittelbar die Einleitung einer medikamentösen antidepressiven Behandlung durch den Notarzt.

B Eine mögliche Suizidalität ist bei entsprechendem Verdacht immer zu explorieren.

C Ein erhöhtes Suizidrisiko besteht bei konkreten Plänen, Vorbereitungen, Suizidversuchen in der Vorgeschichte, Suiziden in der Familie, älteren alleinlebenden Männern.

D Hoffnungslosigkeit und fehlende Zukunftsbezüge weisen oft auf eine akute Suizidalität hin.

E Akute Suizidalität kann mit Fremdgefährdung einhergehen.

\section{Frage 9}

Wodurch ist der stuporöse Zustand gekennzeichnet?

A Stuporöse Patienten bekommen von der Umwelt nichts mit.

B Stuporöse Zustände sind immer hysterischer Natur.

C Stuporöse Patienten können unvermittelt aggressiv werden.

D Stuporöse Zustände lassen sich am besten mit Diazepam behandeln.

E Stuporöse Zustände gibt es ausschließlich bei der wahnhaften Depression.

\section{Frage 10}

Eine der folgenden Aussagen ist falsch. Welche?

A Bewusstseinsstörungen lassen sich hinsichtlich der Quantität und Qualität unterscheiden.

B Eine quantitative physiologische Bewusstseinsstörung ist Schlaf.

C Drogen können zu einer qualitativen Bewusstseinsstörung führen.

D Ein Delir ist eine quantitative Bewusstseinsstörung.

E Psychopharmaka können selbst Ursache psychiatrischer Notfälle sein. 